AperTO - Archivio Istituzionale Open Access dell'Università di Torino

\title{
Relationship between Adult Attachment Patterns, emotional experience and EEG frontal asymmetry
}

\section{This is the author's manuscript}

Original Citation:

Availability:

This version is available http://hdl.handle.net/2318/39178

since

Published version:

DOI:10.1016/j.paid.2007.10.021

Terms of use:

Open Access

Anyone can freely access the full text of works made available as "Open Access". Works made available under a Creative Commons license can be used according to the terms and conditions of said license. Use of all other works requires consent of the right holder (author or publisher) if not exempted from copyright protection by the applicable law. 


\title{
Relationship between adult attachment patterns, emotional experience and EEG frontal asymmetry
}

\author{
Elena Rognoni *, Dario Galati, Tommaso Costa, Manuella Crini \\ Department of Psychology, University of Turin, Via Verdi, 10 - 10124 Torino, Italy
}

Received 5 July 2007; received in revised form 15 October 2007; accepted 16 October 2007

Available online 3 December 2007

\begin{abstract}
This study investigated whether adult attachment styles influence subjective and neurophysiological aspects of emotion. Self-reported emotional arousal and pleasantness and EEG frontal asymmetry were analysed while subjects watched emotional video-clips inducing happiness, fear and sadness with attachmentrelated content. Results showed a clear difference between attachment patterns on emotional arousal, resting frontal asymmetry and fluctuating asymmetry changes. Avoidant individuals responded to positive stimuli with less arousing subjective experience and right frontal asymmetry. In turn, preoccupied individuals showed higher arousal feelings and wider frontal left activation. Opposite patterns were observed in response to fear. These findings support the involvement of attachment in modelling individual emotional response and underlying brain functional processes, accounting partly for individual variability in human emotion. (c) 2007 Elsevier Ltd. All rights reserved.
\end{abstract}

Keywords: Attachment; Frontal asymmetry; EEG; Emotion; Individual difference

\section{Introduction}

Attachment system predisposes infants to seek proximity, support and protection from the attachment figure (Bowlby, 1969/1982): a large body of empirical and theoretical contributions

\footnotetext{
${ }^{*}$ Corresponding author. Tel.: +3390116702923.

E-mail address: rognoni@psych.unito.it (E. Rognoni).
} 
suggest that the different attachment models, shaped by repeated-attachment-caregiving interactions, are involved in regulating cognitive, affective and social functions (for a review, see Cassidy \& Hazan, 1999).

In particular, attachment is strictly linked to emotion: formerly, Bowlby claimed that many of the basic emotions arise during the attachment relationship: fear works as an internal signal to activate attachment motivation and to maintain the current system active; happiness, comfort, and approach-related emotions predisposing the individual to exploration, are associated with satisfying attachment needs and the deactivation of the system; anger sustains complaint behaviours aimed at obtaining the caregiver's attention when not readily available; in case of persisting absence of the caregiver's response, sadness supports attachment deactivation and release of support demand. The individual way to emotionally respond to events could be strongly influenced by attachment experiences possibly affecting neural plasticity which controls the structural and functional organization of innate emotional circuits (Siegel, 1999).

Frontal EEG asymmetry, caused by the relative increase in activity over right or left hemisphere, was identified as a marker of individual differences in functional brain organization (Coan \& Allen, 2004). There is growing evidence that a stable trait-dependent left frontal asymmetry measured at rest and the fluctuating state-dependent left frontal asymmetry in response to emotional stimuli are associated with approach system and positive emotion, whereas right frontal asymmetry is associated with withdrawal system and negative emotion (Davidson, 2004).

Although attachment experiences can strongly impact the individual affective dimension and modulate the underlying neural activity, relatively few studies have considered the relationship between attachment patterns and frontal asymmetry. Early studies investigated frontal asymmetry in infancy during maternal separation- and reunion-like interactions: infants who cried in response to separation showed greater right frontal than those who did not cry as a stable trait (Davidson \& Fox, 1989; Fox, Bell, \& Jones, 1992), as well as a state-dependent response (Fox \& Davidson, 1988; Buss et al., 2003), while the left prefrontal activity increased during motherapproach experience (Fox \& Davidson, 1987). Cohen and Shaver (2004) investigated hemispheric asymmetries associated with adult attachment, finding that avoidant individuals experienced positive emotion less intensely and exhibited a disadvantage in processing positive attachment-related information in the right hemisphere.

This work aimed at investigating the subjective and neurophysiological aspects of emotion associated with adult attachment models. The film-induced emotions of happiness, fear, and sadness were examined, for their strict relationship with attachment activation and deactivation, evaluating the self-reported emotional arousal and pleasantness, and EEG frontal asymmetry, measured both at rest and in response to emotional stimuli.

The hypotheses of this study were based on the desirability of bridging contributions from the attachment theory with findings on the relationship between frontal asymmetry and individual affective style (Davidson, 2004). Attachment groups were defined in accordance with the model of adult attachment by Bartholomew and Horowitz (1991), who identified four main clusters: secure-free, preoccupied, dismissing-avoidant and fearful-avoidant. It was considered that, although attachment patterns can be split into two main clusters, secure and insecure, the three insecure types differ from each other for several aspects, including internal working models and affective styles. Hence, we expected differences between the four types and the three insecure types, and not only between secure and insecure. The greatest difference was expected between preoccupieds 
and avoidants, due to the opposite ways in which they learned to regulate attachment activationdeactivation. Avoidant subjects tend to hypo-activate attachment, so as to attain the adaptive outcome of suppressing attachment signals and giving up to seek the support they have often experienced to have been denied. In adulthood, avoidant individuals tend to withdraw interpersonal intimacy and to exhibit reduced approach behaviour in attachment-related contexts, tend to experience and express less intensely positive emotion (Searle \& Meara, 1999), rate social interaction as boring and less engaging (Tidwell, Reis, \& Shaver, 1996) and inhibit attachment representations in attachment-related conditions (Mikulincer, Gillath, \& Shaver, 2002).

In turn, preoccupied individuals in infancy experienced incoherent and unpredictable caregiving responses, acquiring adaptive strategies to improve the possibility to achieve and maintain proximity of the caregiver. These strategies mainly consist in increasing attachment signals and are supported by hyper-activation of attachment; preoccupied adults tend to intensely express fear, anxiety and anger when faced with separation, loneliness and threat (Bartholomew \& Horowitz, 1991).

As regards these attachment-related themes it was expected that, compared to other attachment patterns, avoidance showed (a) less arousal and less pleasant subjective emotion in response to positive video-clips of happiness, (b) right-sided frontal activity as trait-dependent baseline, and (c) lower relative left frontal asymmetry in response to positive stimuli. In turn, preoccupied individuals were expected to show (a) higher self-reported arousal and lower pleasantness in response to negative emotions, in particular fear, (b) trait-dependent right frontal asymmetry, and (c) greater right asymmetry during negative emotion, in particular fear.

\section{Method}

\subsection{Participants}

Thirty-nine healthy, right-handed (measured by Edinburgh Handedness Inventory, Oldfield, 1971), volunteers took part in the experiment (20 women, 19 male, mean age $=24.36$, $\mathrm{SD}=2.48$ ). A preliminary interview was conducted by a clinician to select neurologically and psychiatrically well, unmedicated subjects. Then, subjects filled the four self-report scales used to assess attachment as descript below. Only subjects who received the same classification in all the scales were included in the experiment: 9 subjects were classified as avoidant ( 4 females), 14 frees ( 8 females), 9 preoccupieds ( 4 females), and 7 fearful-avoidants ( 3 females). The groups did not statistically differ on number of men and women, Fisher's exact test $=.77, p=.92$, while they tended to differ on age, $F(3,31)=2.7, p=.06$.

The study was approved by the Ethics Committee of the University of Turin, in accordance with "the Ethical Principles of Psychologists and Code of Conduct" (APA, 2002).

\subsection{Materials}

\subsubsection{Film stimuli}

Stimuli consisted of 48 video-clips with emotional content of happiness, sadness, fear and neutral content (12 film clips for each condition), extracted from a set of stimuli prepared to induce 
pure basic emotions and pre-tested on a sample of judges who rated the emotional meaning of sequences. For the present study, we selected those stimuli that depicted attachment-related scenarios: the video-clips of happiness depicted lovers' encounters, pleasant social interactions, close relationships, familiar reunions, affective tender exchanges. Sadness sequences depicted scenes of loss, death, abandon, sorrow, and loneliness. Fear sequences depicted dangerous situations, and, mostly, interpersonal threats. Neutral sequences depicted a series of routine actions. All the stimuli were $10 \mathrm{~s}$ long, colour, without sound and balanced in relation to the presence of human beings.

\subsubsection{Attachment assessment}

The attachment patterns were assessed using different self-report scales designed to evaluate the attachment models in adult close relationships and diffusely employed in attachment research on adulthood. Although there has been some disagreement, the validity and reliability of these instruments have received support by a large and growing literature (for a critical review, see Shaver $\&$ Mikulincer, 2002).

The reference scale was the Relationship Questionnaire (RQ, Bartholomew \& Horowitz, 1991), followed by the Experiences in Close Relationships-Revised Questionnaire (ECR-R, Fraley, Waller, \& Brennan, 2000), the Adult Attachment Scale (Collins \& Read, 1990), and the Parenting Bonding Instrument (Parker, 1990).

\subsubsection{Self-report of emotional experience}

The subjective emotional response to film stimuli was assessed through a self-report scale consistent with dimensional models of emotion, including a 9-point Likert scale for pleasantness (from 1 indicating very unpleasant emotion, to 9, very pleasant) and arousal (from 1 indicating a calm state, to 9 , very excited).

\subsubsection{Recordings apparatus}

EEG was recorded from 19 scalp sites (Fp1, Fp2, F7, F8, F3, F4, Fz, C3, C4, Cz, T3, T4, T5,T6, P3, P4, Pz, O1, O2), referenced to a ground electrode attached to the center of the forehead. A QuickCup was used with embedded $\mathrm{Ag} / \mathrm{AgCl}$ electrodes and ElectroCap Gel. Vertical and horizontal electrooculogram (EOG) was measured to control for ocular artefacts using $\mathrm{Ag} / \mathrm{AgCl}$ electrodes, placed $1 \mathrm{~cm}$ above and below the middle of right eye, and as close as possible to the left and right outer canthi of the eyes. The impedance was ever kept below the threshold of $5 \mathrm{~K} \Omega$. EEG and EOG signals were amplified by a multi-channel bio-signal amplifier (bandpass $0.3-70 \mathrm{~Hz}), \mathrm{A} / \mathrm{D}$ converted at $256 \mathrm{~Hz}$ per channel with 12-bit resolution.

\subsection{Procedure}

After obtaining written approval, subjects took part in the preliminary anamnesis interview. At the end, they completed the attachment scales, alternated with masking tests used to avoid the exclusive concentration on close relationships. About two weeks after, subjects were met in the EEG laboratory. They were told that the study investigated individual differences in brain response to visual communication of movies and that the only task demand was to pay attention to the film. Prior and after the experiment, two eye-open baselines of five minutes was taken. 
The stimuli were presented in a block design with a regular baseline (black screen). The experiment included four blocks of three stimuli per each experimental condition (neutral, happiness, sadness and fear), presented in two runs separated by a resting period. Stimuli were assigned to blocks and runs in randomized order both between and within subjects, as the block in each run, with the restriction that two blocks per condition were presented in a single run, and that no more than one block of the same condition was repeated in succession.

In order to avoid any interference with EEG recordings, subjective ratings were collected at the end of the experimental runs while participants watched for a second time the video-clips in randomized order.

\section{Data analysis and results}

\subsection{EEG pre-processing and frontal asymmetry estimate}

The epoched EEG traces (1 s each) were band-pass filtered with a forward reverse filter. Artefacts were eliminated off-line from EEG recordings by submitting the data to ICA pre-processing (Independent Component Analysis, Jung et al., 1998), which removes a wide variety of EEG artefacts, separating the EEG and its artefacts into independent components, without necessarily relying on reference channels for each type of artifact. This avoids the problem of potential mutual contamination of regressing and regressed channels. Through a careful visual inspection the components expressing EOG contributions to EEG and further artefacts (muscular movements or heart rate) were removed. About $10 \%$ of EEG data were excessively contaminated with artefacts and rejected, with no difference among groups.

A fast Fourier transform (FFT), using a Hamming window, was used for estimates of spectral power $\left(\mu \mathrm{V}^{2}\right)$. Power values within each epoch were averaged, converted to power density $\left(\mu \mathrm{V}^{2} /\right.$ $\mathrm{Hz})$ and grouped into alpha $(8-12 \mathrm{~Hz})$ frequency band.

Asymmetry scores were computed following the most commonly used approach (Coan \& Allen, 2004) that consists of subtracting the natural log of left hemisphere alpha power from the natural $\log$ of right hemisphere alpha power (Ln Right - Ln Left). This method provides a one-dimensional scale representing the relative activity of the right and left hemisphere. In interpreting this scale, it has to be taken into consideration that power in alpha band is inversely related to neural processing, so that decreases in alpha power indicate increases in the cortical activation. Hence, the middle score on the frontal asymmetry measure equalling zero, indicates symmetrical activity, negative scores reflect greater relative right-sided activity, and positive scores reflect greater left activity.

\subsection{Subjective results}

The pleasantness and arousal ratings were subjected to three-way ANOVA, including the within-subjects factor Condition (four levels: neutral, happiness, fear, sadness), and the between-subjects factors Attachment (four levels: avoidant, free, preoccupied and fearful/avoidant) and Sex. Results are reported in Table 1. The main effects of Condition and Attachment were examined using Bonferroni-corrected post hoc comparisons. Neutral showed lower arousal ratings than 
Table 1

Statistical significance of ANOVA on self-reported arousal and pleasantness

\begin{tabular}{|c|c|c|c|c|}
\hline Source & Measure & $F(\mathrm{df})$ & $p$ & $\eta^{2}$ \\
\hline \multirow[t]{2}{*}{ Condition } & Arousal & $83.61(3,84)$ & .000 & .75 \\
\hline & Pleasantness & $108.81(2.13,59.56)$ & .000 & .80 \\
\hline \multirow[t]{2}{*}{ Attachment } & Arousal & $5.26(3,28)$ & .005 & .36 \\
\hline & Pleasantness & $2.12(3,28)$ & n.s. & \\
\hline \multirow[t]{2}{*}{ Condition $\times$ Attachment } & Arousal & $3.02(9,84)$ & .004 & .25 \\
\hline & Pleasantness & $.87(6.38,59.56)$ & n.s. & \\
\hline
\end{tabular}

emotion, $p<.001$, and happiness scored significantly higher pleasantness compared to all the other conditions, $p<.001$, confirming that stimuli were suitable for inducing expected emotion. As regards the Attachment effect, preoccupieds subjectively responded with higher arousal compared to fearful-avoidant, $p<.01$, and avoidant, $p=.051$. More interestingly, the significant interaction effect of Condition $\times$ Attachment indicates that attachment groups reported different arousal ratings according to emotion (Fig. 1). The same analysis was performed to test the effect of attachment security vs. insecurity, grouping the insecure categories, but no significant result emerged.

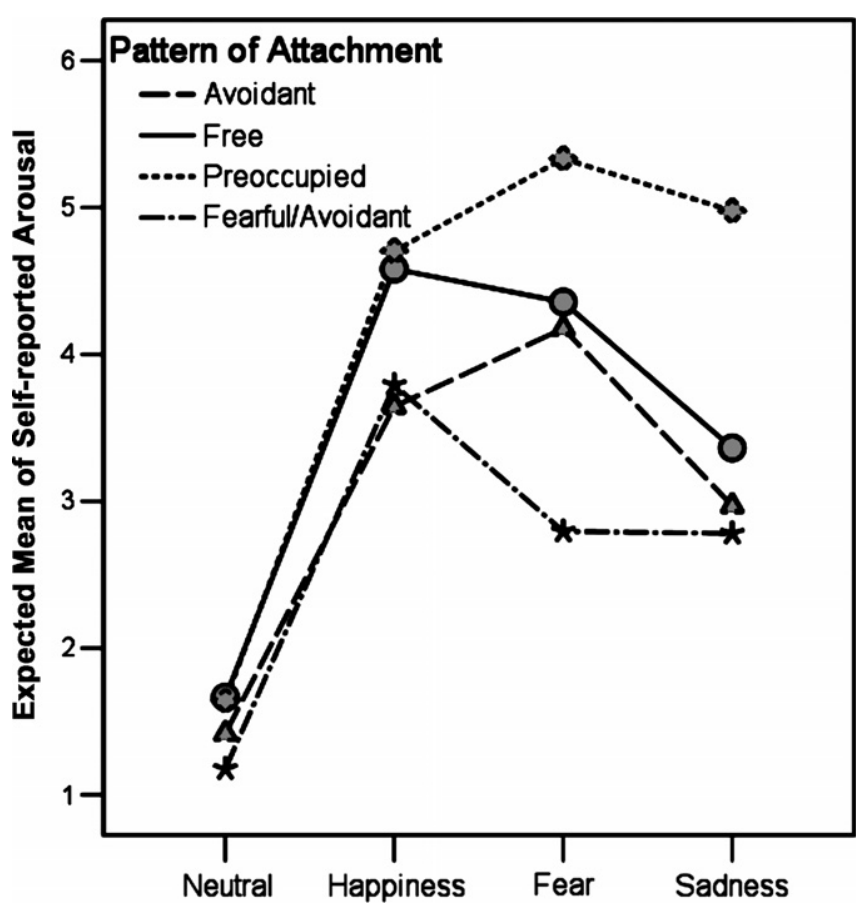

Fig. 1. Interaction effect between condition and attachment on self-reported arousal. 


\subsection{Frontal asymmetry results}

Data for frontal asymmetry during stimulation and rest (Fig. 2) were analysed by repeated ANOVA including Condition (five levels: rest, neutral, happiness, fear and sadness), Attachment and Sex. Age was included as covariate considering the almost significant difference found between groups, but no significant effects emerged, so the covariate was removed from the model. As the sphericity assumption was violated, the Huynh-Feldt correction to degrees of freedom was used. Analysis turned out to be significant for Condition, $F(4,124)=6.03, p<.001, \eta^{2}=.16$, indicating that distinct emotions were associated to specific frontal activation patterns: in accordance with previous findings, happiness induced greater left activation, while the two negative emotions induced greater right activation. More importantly, the significant interaction effect of Condition $\times$ Attachment, $F(12,124)=7.2, p<.001, \eta^{2}=.41$, indicates that these emotion-specific patterns differed among the attachment groups (Fig. 3).

The interaction effect (Condition $\times$ Attachment) was not found over the posterior regions (parietal and occipital), $F(7.07,73.04)=1.02, p<.43$, in accordance with previous studies supporting the importance of the frontal region in regulating affective behaviour.

The analysis on security vs. insecurity failed to reach significance level, whereas significant Pearson correlations emerged between the continuous measures of the attachment scale (RQ) and the asymmetry ratings for the different conditions: during happiness response, higher scores on avoidance scale were associated with greater right asymmetry, $r=-.42, p<.01$, and higher scores on
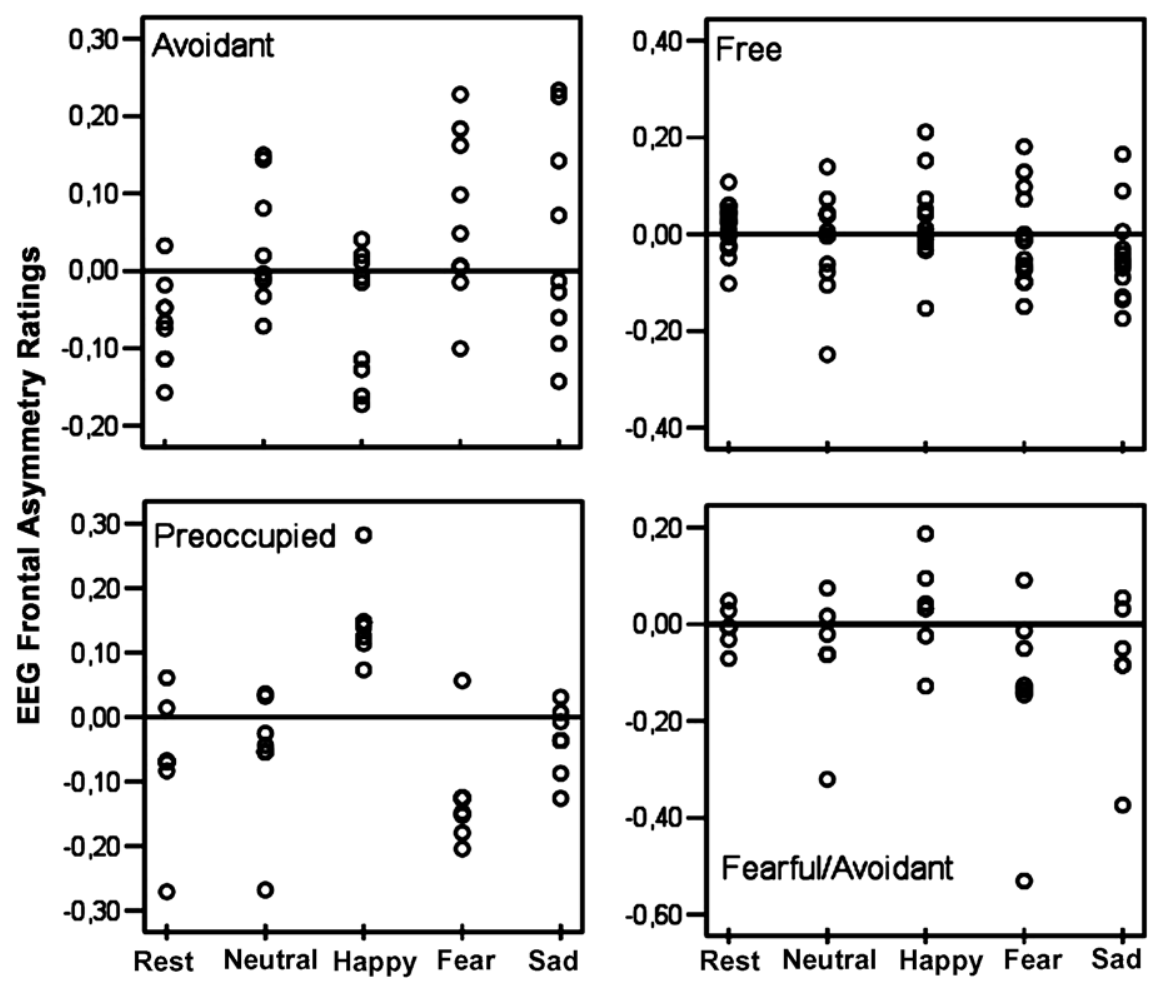

Fig. 2. Scatterplots of frontal asymmetry exhibited by subjects in each attachment group and condition. 


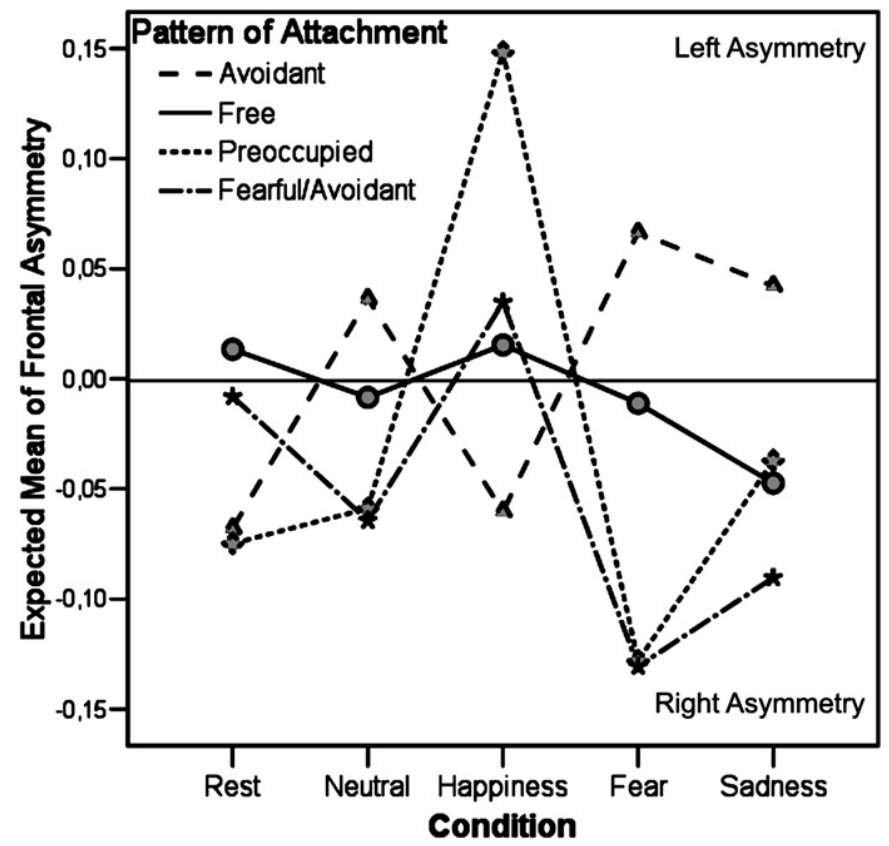

Fig. 3. Interaction effect between condition and attachment on frontal asymmetry.

the preoccupied scale were associated with greater left asymmetry, $r=.41, p<.01$; during fear, higher scores on preoccupied and fearful-avoidance scales correlated with greater right activation, $r=-.38, p<.05, r=-.38, p<.05$, respectively. In addition, avoidance scores was associated with greater right rest activity, $r=-.35, p<.05$, and free scale showed an almost significant correlation with greater left activity, $r=.29, p=.08$.

\subsection{Crossing subjective and EEG data}

Partial Pearson correlations were performed, removing repeated effects of subjects, between self-reported scores of arousal and pleasantness and asymmetry scores across all experimental conditions: pleasantness turned out to positively correlate with frontal asymmetry across groups, $r=.22, p<.01$, confirming that higher pleasantness was associated with greater left prefrontal activity.

Analysis within each group revealed that pleasantness and frontal asymmetry correlated positively in preoccupied individuals, $r=.71, p<.001$. Oppositely, a negative correlation was found in avoidants, $r=-.45, p<.05$, indicating that higher subjective pleasantness was associated with lower left activity and relatively higher right activity.

\section{Discussion}

Concerning the subjective component of emotion, the hypothesis of attachment-related differences was confirmed for arousal, but not for pleasantness. The security-insecurity dimension of 
attachment did not show any difference, supporting the theory that the three insecure categories are characterized by qualitatively specific emotional experience. Interestingly, only responses to emotional stimuli differed among attachment patterns, while subjective response to neutral stimuli was similar. In particular, the subjective emotional experience of avoidant and preoccupied subjects differed significantly, in line with hypotheses.

For preoccupied individuals the experience of negative emotions of fear and sadness was more arousing. Consistent with the attachment theory, the intense experience of fear or distress associated with attachment hyper-activation can lead to effectively communicate that a heavy threat is present, and therefore the caregiver's attention is more likely to be captured.

In turn, during positive emotion preoccupied subjects exhibited a level of emotional arousal similar to that of free subjects and higher than that of avoidants and fearful-avoidants. On the contrary, avoidant individuals seemed to experience positive emotions as less arousing than other groups: in accordance with previous findings (Cohen \& Shaver, 2004), this result suggests that avoidance strategies may minimize the impact of positive emotions associated with satisfaction of attachment needs which were rarely fulfilled in their attachment experiences.

Differences among attachment patterns were also found for the neurophysiological aspect of emotion, investigated through frontal asymmetry. Trait-dependent frontal activity associated with security turned out to be sufficiently symmetrical, tending toward higher left asymmetry. In turn, avoidants and preoccupieds showed a significantly lower asymmetry rating indicating a greater right resting activity. These results are in agreement with the literature, pointing to individuals disposed to approach-related behaviour and positive affection, such as free individuals could be (Simpson, Collins, Tran, \& Haydon, 2007), exhibiting higher left frontal activity, whilst the opposite pattern is associated with a disposition for withdrawal-related behaviour and negative affection (Davidson, 2004) which could occur with attachment insecurity. Indeed, as confirmed by this analysis on subjective response, preoccupied subjects tended to experience stronger negative emotions and avoidant subjects experienced weaker positive emotions.

Higher right resting asymmetry had already been found in children who exhibited inhibited behaviour in social interactions, whereas social competence was related to greater left activity (Fox et al., 1995). These patterns could be paired with the present trends in adult attachment, considering the influence of internal models on social behaviour: avoidant children are more likely to retire from social interactions, whereas preoccupied children could display social anxiety and timidity when facing unfamiliar or novel places. Differently, the insecure fearful/avoidant group exhibited a relatively symmetrical resting pattern similar to frees, although it tended toward right asymmetry. This unexpected result might be interpreted in line with the theory proposed by Main (1991), according to which disorganized attachment is characterized by multiple and incompatible internal models, which could predispose individuals to activate at the same level both the approach and withdrawal motivations. In other terms, fearful/avoidants may tend to activate the approach system more than the other insecure-attached individuals, exhibiting a relatively greater left resting asymmetry.

In response to emotion, frontal asymmetry was found to be specifically related to attachment. In response to happiness, free, fearful/avoidant and especially preoccupied subjects displayed an increased frontal asymmetry score, pointing to higher left activation in agreement with previous evidence of the functional role of the left prefrontal cortex in approach-related emotions. In contrast, a completely opposite pattern was observed in avoidants, who responded to positive stimuli 
with an increased right activation. The lower level of arousal reported by avoidants in response to happiness stimuli suggests that these subjects restrictively experience positive affect, and arguably this subjective aspect could be related to lower involvement of the left hemisphere in processing positive information and/or to increased right activation. Also, the higher left frontal asymmetry of preoccupied subjects in a state of happiness may be matched by their higher subjective response: contrary to that of avoidant individuals, this pattern of attachment accentuates the desire for intimacy and proximity, while expressing high distress in expectancy of loneliness, separation or abandonment. Our happiness video-clips depicting interpersonal affective exchange could be processed as highly pleasant and desirable stimuli by preoccupied subjects, channelling a large amount of neural resources associated with the approach system. Conversely, avoidant attachment models could modulate the processing of these same stimuli showing intimacy by activation of neural correlates of the withdrawal system. Interestingly, fearful-avoidants showed a left-sided pattern similar to preoccupieds and frees, consistently with the strong desire for interpersonal intimacy characterizing this attachment style (as also described in the RQ scale).

Frontocortical responses to negative emotion produced completely different patterns: preoccupied subjects displayed lower frontal asymmetry, pointing to higher activation over the right hemisphere, especially for fear, whereas avoidant individuals displayed stronger activation of the left hemisphere. The highly enhanced right activation shown by preoccupied subjects is coherent with their self-reported high arousal in response to fear, and is in line with previous findings: children exhibiting fearful or shy behaviour, generally present in resistant-ambivalent attachment, have been observed to have greater right frontal EEG activity at rest (Calkins, Fox, \& Marshall, 1996), as well as during stressful tasks (Schmidt, Fox, Schulkin, \& Gold, 1999) and separation (Buss et al., 2003). Additionally, Fox and Davidson (1988) observed that during maternal separation infants who displayed anger and sadness, but did not cry, showed greater left frontal activation, whereas infants who cried showed greater right asymmetry. Similar findings suggest that right frontal asymmetry could be associated with intense and full expression of separation distress, as expected in resistant infants and preoccupied adults, whereas left asymmetry could be associated with the inhibition of attachment behaviour like crying, which is typical of attachment avoidance.

From these results it emerged that avoidant individuals process unpleasant stimuli as less arousing, activating approach-related neural circuits or deactivating withdrawal-related circuits, depending on attachment hypo-activation. Also, they tend to process pleasant stimuli related to intimacy and close relationships as less arousing, activating the withdrawal neural circuits. Preoccupied subjects showed the opposite tendency, enhancing withdrawal circuit activation in a state of fear, and stimulating the approach circuits in response to happiness. This interpretation is further supported by significant resulting correlations. In fact, as the emotion was pleasant, frontal asymmetry increased in preoccupied subjects (right asymmetry), and decreased in avoidant individuals (left asymmetry).

The fearful/avoidant group showed further specific frontal asymmetry changes, which point to the attachment theory. During fear response fearful/avoidant subjects exhibited an interesting mismatch between self-reported emotional arousal and frontal asymmetry changes, resulting in the feeling of fear being less arousing, with consequent lower ratings compared to the other groups and to all the other emotions; nonetheless, they largely activated the right hemisphere, just as preoccupied subjects did. This supports the theory that attachment experiences of the subjects 
involved have impacted and modelled the fear system through repeated interpersonal exchanges with a frightening-frightened caregiver: in these conditions, infants are unable to construct integrated and coherent internal models for self and others (Main, 1991). According to Main, these multiple and incompatible representations cause excessive loading of both cognitive-emotional system and working memory, becoming less accessible to the awareness level, so that metacognitive monitoring of such representations becomes difficult. Several clinical, theoretical and experimental contributions suggested that the disorganization of attachment could involve metacognitive disfunctioning, especially in emotionally laden situations (Simpson \& Rholes, 2002).

Finally, sex influence merits comment given the growing evidence for gender differences on frontal lateralization and affective behaviour. Although not significant, trends showed more lateralized patterns in men, independently on attachment and condition, in accordance with previous findings (e.g., Wager, Phan, Liberzon, \& Taylor, 2003), suggesting to further investigate gender effects.

This study is somewhat limited, as first by the small and inhomogeneous number of subjects within each group. All the same, the effects turned out to be statistically significant and in line with our hypotheses and the predictions of the attachment theory, encouraging further investigations. Second, frontal asymmetry metric can account for relative change in right and left frontal regions, but cannot account for associated contributing factors, which can only be guessed. However, it makes for simplification of analyses involving correlation and statistical tests on the effects of other individual variables (Coan \& Allen, 2004). Future research using a larger sampling base could expand the scope of this topic.

The findings of this study demonstrate that individual differences in emotional responses, at both subjective and neurophysiological level, are partly a function of individual attachment models, supporting the relationship between attachment, emotion and cerebral activity in adults. Differences in emotional experience were consistent with the way individuals with distinct attachment models behave in their close relationships and in certain emotionally laden situations. On this basis, it is suggested that investigation in attachment models, which plays a key role in regulating emotional systems, could make a significant contribution toward understanding the complexity of human emotion, as well as the processes through which experience influences the neural organization of affective systems.

\section{References}

American Psychological Association (2002). Ethical principles of psychologists and code of conduct. American Psychologist, 57, 1060-1073.

Bartholomew, K., \& Horowitz, L. M. (1991). Attachment styles among young adults: A test of a four-category model. Journal of Personality and Social Psychology, 61(2), 226-244.

Bowlby, J. (1982). Attachment and loss (Vol. 1). New York: Basic, 1969 pp.

Buss, K. A., Schumacher, J. R., Dolski, I., Kalin, N. H., Goldsmith, H. H., \& Davidson, R. J. (2003). Right frontal brain activity, cortisol, and withdrawal behavior in 6-month-old infants. Behavioral Neuroscience, 117(1), 11-20.

Calkins, S. D., Fox, N. A., \& Marshall, T. R. (1996). Behavioral and physiological antecedents of inhibition in infancy. Child Development, 67, 523-540.

Cassidy, J., \& Hazan, P. R. (1999). Handbook of attachment. New York: Guilford Press. 
Coan, J. A., \& Allen, J. J. B. (2004). Frontal EEG asymmetry as a moderator and mediator of emotion. Biological Psychology, 67, 7-49.

Cohen, M. X., \& Shaver, P. R. (2004). Avoidant attachment and hemispheric lateralization of the processing of attachment- and emotion-related words. Cognition \& Emotion, 18(6), 799-813.

Collins, N. L., \& Read, S. J. (1990). Adult attachment, working models, and relationship quality in dating couples. Journal of Personality and Social Psychology, 58, 644-663.

Davidson, R. J. (2004). What does the prefrontal cortex "do" in affect: perspectives on frontal EEG asymmetry research. Biological Psychology, 67, 219-233.

Davidson, R. J., \& Fox, N. A. (1989). Frontal brain asymmetry predicts infants' response to maternal separation. Journal of Abnormal Psychology, 98, 127-131.

Fox, N. A., Bell, M. A., \& Jones, N. A. (1992). Individual differences in response to stress and cerebral asymmetry. Developmental Neuropsychology, 8, 161-184.

Fox, N. A., \& Davidson, R. J. (1987). Electroencephalogram asymmetry in response to the approach of a stranger and maternal separation in 10-month-old infants. Developmental Psychology, 23, 233-240.

Fox, N. A., \& Davidson, R. J. (1988). Patterns of brain electrical activity during facial signs of emotion in ten month old infants. Developmental Psychology, 24, 230-236.

Fox, N. A., Rubin, K. H., Calkins, C. D., Marshall, T. R., Coplan, R. J., Porges, S. W., et al. (1995). Frontal activation asymmetry and social competence at four years of age. Child Development, 66, 1770-1784.

Fraley, R. C., Waller, N. G., \& Brennan, K. A. (2000). An item response theory analysis of self-report measures of adult attachment. Journal of Personality \& Social Psychology, 78(2), 350-365.

Jung, T.-P., Humphries, C., Lee T.-W., Makeig, S., McKeown, M. J., Iragui, V., \& Sejnowski, T. J. (1998). Extended ICA removes artifacts from electroencephalographic recordings. In D. Touretzky, M. Mozer, \& M. Hasselmo (Eds.), Advances in neural information processing systems (Vol. 10, pp. 894-900).

Main, M. (1991). Metacognitive knowledge, metacognitive monitoring, and singular (coherent) vs. multiple (incoherent) model of attachment: Findings and directions for future research. In C. M. Parkes, J. StevensonHinde, \& P. Marris (Eds.), Attachment across the life cycle (pp. 127-159). London: Tavistockl Routledge.

Mikulincer, M., Gillath, O., \& Shaver, P. R. (2002). Activation of the attachment system in adulthood: Threat-related primes increase the accessibility of mental representations of attachment figures. Journal of Personality and Social Psychology, 83(4), 881-895.

Oldfield, R. C. (1971). The assessment and analysis of handedness: The Edinburgh Inventory. Neuropsychologia, 6, $97-113$.

Parker, G. (1990). The parental bonding instrument: A decade of research. Social Psychiatry and Psychiatric Epidemiology, 25(6), 281-282.

Schmidt, L. A., Fox, N. A., Schulkin, J., \& Gold, P. W. (1999). Behavioral and psychophysiological correlates of selfpresentation in temperamentally shy children. Developmental Psychobiology, 35, 119-135.

Searle, B., \& Meara, N. M. (1999). Affective dimensions of attachment styles: Exploring self-reported attachment style, gender, and emotional experience among college students. Journal of Counseling Psychology, 46(2), 147-158.

Shaver, P. R., \& Mikulincer, M. (2002). Attachment-related psychodynamics. Attachment and Human Devolopment, 4, 133-161.

Siegel, D. J. (1999). The developing mind. New York: Guilford.

Simpson, J. A., Collins, W. A., Tran, S., \& Haydon, K. C. (2007). Attachment and the experience and expression of emotions in romantic relationships: A developmental perspective. Journal of Personality and Social Psychology, 92(2), 355-367.

Simpson, J. A., \& Rholes, W. S. (2002). Fearful-avoidance, disorganization, and multiple working models: Some directions for future theory and research. Attachment \& Human Development, 4(2), 223-229.

Tidwell, M. C., Reis, H. T., \& Shaver, P. R. (1996). Attachment, attractiveness, and social interaction: A diary study. Journal of Personality and Social Psychology, 71(4), 729-745.

Wager, T. D., Phan, K. L., Liberzon, I., \& Taylor, S. F. (2003). Valence, gender, and lateralization of functional brain anatomy in emotion: A meta-analysis of findings from neuroimaging. NeuroImage, 19, 513-531. 